\title{
ON THE SHARP GROWTH OF ANALYTIC CAUCHY-STIELTJES TRANSFORMS
}

\author{
By D. J. Hallenbeck and K. SamotiJ*
}

\section{Introduction}

Let $\Delta=\{z:|z|<1\}$ and $\Gamma=\{z:|z|=1\}$. Let $\mathscr{M}$ denote the set of complexvalued Borel measures on $\Gamma$. For each $\alpha \geqq 0$ the family $\mathscr{T}_{\alpha}$ of functions analytic in $\Delta$ is defined as follows. If $\alpha>0$ then $f \in \mathscr{F}_{\alpha}$ provided that there exists $\mu \in$ $\mathscr{M}$ such that

$$
f(z)=f_{\mu}(z)=\int_{\Gamma} \frac{1}{(1-\bar{\zeta} z)^{\alpha}} d \mu(\zeta)
$$

for $|z|<1$. Also, $f \in \mathscr{F}_{0}$ provided that there exists $\mu \in \mathscr{M}$ such that

$$
f(z)=f_{\mu}(z)=\int_{\Gamma} \log \frac{1}{(1-\bar{\zeta} z)} d \mu(\zeta)+f(0)
$$

for $|z|<1$ (Here and throughout this paper every logarithm means the principal branch.). The classes $\mathscr{F}_{\alpha}$ for $\alpha \geqq 0$ were first studied in [3] and [4]. Of course, the case $\alpha=1$ is classical and well studied in the literature. The mapping from $\mathscr{M}$ to $\mathscr{F}_{\alpha}$ given by $\mu \rightarrow f_{\mu}$ is not one-to-one, i.e., the correspondence between measures and functions in $\mathscr{T}_{\alpha}$ is not unique. Suppose that $\mu \in \mathscr{M}$. Let $|\mu|$ denote the total variation norm of $\mu$ and let $\|\mu\|=|\mu|(\Gamma)$. For $|\zeta|=1$ and $0<$ $x \leqq \pi$ let $I(\zeta, x)$ denote the closed arc on $\Gamma$ centered at $\zeta$ and having length $2 x$. A function $w$ is defined on $[0, \pi]$ by

$$
w(x)=|\mu|(I(\zeta, x)) \quad \text { for } \quad 0<x \leqq \pi \text { and } \quad w(0)=0 .
$$

To indicate the dependence of $w$ on $\zeta$ and $x$ we sometimes write $w(x)=w(x, \xi$, $\mu)$ or $w(x)=w(x, \mu)$. As explained in [1] formula (1) is equivalent to

$$
f(z)=\int_{-\pi}^{\pi} \frac{1}{\left(1-e^{-i t} z\right)^{\alpha}} d g(t)
$$

where $g$ is a complex-valued function of bounded variation on $[-\pi, \pi]$. Similar remarks apply to (2). We point out, that in the standard way, our measures may be regarded as being defined on $[-\pi, \pi]$ rather than on $\Gamma$. This is noth-

* This research was partially supported by a grant from Komitet Badan Naukowych.

Received December 15, 1992; revised November 29, 1993. 
ing more than a technical convenience which we will use in Theorems 1 and 2.

In [1] and [2] the authors examined the interplay between local and global aspects of radial and nontangential limits for functions in $\mathscr{F}_{\alpha}(\alpha \geqq 0)$ and how this depends on an analysis of properties of the representing measures.

In this paper we intend to describe precisely in what sense the following two theorems from [1] and [2] respectively can be said to be sharp.

THEOREM A. Let $\alpha \geqq 0$. Suppose that $f \in \mathscr{F}_{\alpha}$ and (1) or (2) holds where $\mu$ $\in M$. Let $w$ be defined by (3) where $\zeta=e^{i \theta}$ and $-\pi \leqq \theta \leqq \pi$. Then there are positive constants $A$ and $B$ depending only on $\alpha$ such that

$$
\left|f\left(r e^{i \theta}\right)\right| \leqq A\|\mu\|+B \int_{1-r}^{\pi} \frac{w(x)}{x^{\alpha+1}} d x \quad \text { for } \quad 0 \leqq r<1 .
$$

THEOREM B. Suppose that $\alpha \geqq 1, g$ is a complex-valued function of bounded variation on $[-\pi, \pi]$ and let

$$
f(z)=\int_{-\pi}^{\pi} \frac{1}{\left(1-e^{-i t} z\right)^{\alpha}} d g(t)
$$

for $|z|<1$. Assume that $g$ is differentiable at some $\theta$ in $[-\pi, \pi]$. If $\alpha>1$ then $\left(1-e^{-i \theta} z\right)^{\alpha-1} f(z)$ has the nontangential limit zero at $e^{i \theta}$. If $\alpha=1$ then $f(z) / \log \left(1 /\left(1-e^{-i \theta} z\right)\right)$ has the nontangential limit zero at $e^{i \theta}$.

In Theorems 1 and 2 to follow we show that Theorem A [1] is sharp and in Theorem 3 we show that Theorem B [2] is sharp. We note that when $1 \leqq$ $\alpha \leqq 2$, Theorem B was shown to be "sharp" in [Theorem 5,1$]$.

In our Theorem 3 we strengthen this result by replacing "lim sup" by "lim" and extend the range of $\alpha$ to all $\alpha \geqq 1$. This result for $\mathscr{F}_{\alpha}$ classes is analogous to the result of G. D. Taylor [5] for $H^{p}$ spaces.

Proposition 1. Let $\alpha>0$ and $\mu$ be a non-negative measure on $[-\pi, \pi]$. Then

$$
\frac{\min \left(\alpha, \pi^{-\alpha}\right)}{2^{\alpha / 2}}\left[\|\mu\|+\int_{1-r}^{\pi} \frac{w(x)}{x^{\alpha+1}} d x\right] \leqq \int_{-\pi}^{\pi} \frac{d \mu(t)}{\left|1-r e^{-i t}\right|^{\alpha}}
$$

and

$$
\int_{-\pi}^{\pi} \frac{d \mu(t)}{\left|1-r e^{-i t}\right|^{\alpha}} \leqq A\|\mu\|+B \int_{1-r}^{\pi} \frac{w(x)}{x^{\alpha+1}} d x
$$

where $w(x)=w(x, 1, \mu)$ and $r \in[0,1)$. Also the constants $A$ and $B$ depend only on $\alpha$.

Proof. The proof of (5) can be found in [1]. To prove (4) we first remark that it is easy to use the identity $\left|1-r e^{2 x}\right|^{2}=(1-r)^{2}+4 r \sin ^{2}(x / 2)$ and the inequality $\sin |x| \leqq|x|$ to prove that

$$
\frac{1}{\left|1-r e^{2 x}\right|}>\frac{1}{\sqrt{2}} \frac{1}{1-r}
$$


when $x \in I_{1}=[-(1-r), 1-r]$, and that

$$
\frac{1}{\left|1-r e^{2 x}\right|}>\frac{1}{\sqrt{2}} \frac{1}{|x|}
$$

when $x \in I_{2}=[-\pi, \pi] \backslash I_{1}$.

It follows from (6) and (7) that

$$
\begin{aligned}
\int_{-\pi}^{\pi} \frac{d \mu(t)}{\left|1-r e^{-i t}\right|^{\alpha}} & >\frac{1}{2^{\alpha / 2}} \frac{1}{(1-r)^{\alpha}} \int_{I_{1}} d \mu(t)+\frac{1}{2^{\alpha / 2}} \int_{I_{2}} \frac{1}{|t|^{\alpha}} d \mu(t) \\
& =\frac{1}{2^{\alpha / 2}} \frac{w(1-r)}{(1-r)^{\alpha}}+\frac{1}{2^{\alpha / 2}} \int_{1-r}^{\pi} \frac{d w(x)}{x^{\alpha}} .
\end{aligned}
$$

An integration by parts gives

$$
\int_{1-r}^{\pi} \frac{d w(x)}{x^{\alpha}}+\frac{w(1-r)}{(1-r)^{\alpha}}=\frac{w(\pi)}{\pi^{\alpha}}+\alpha \int_{1-r}^{\pi} \frac{w(x)}{x^{\alpha+1}} d x .
$$

From (8), (9) and the fact that $w(\pi)=\|\mu\|$ we infer that (4) holds.

LEMma 1. Suppose $0<\alpha<2$. Then we have

$$
\operatorname{Re} \frac{e^{i \operatorname{sgn}(t)(\pi \alpha / 4)}}{\left(1-r e^{-i t}\right)} \geqq \cos \left(\frac{\pi \alpha}{4}\right) \frac{1}{\left|1-r e^{-i t}\right|^{\alpha}}
$$

whenever $t \in[-\pi, \pi]$ and $r \in[0,1)$.

Proof. Note that since both sides of (10) are even functions of $t$, it is enough to prove the lemma for $0<t \leqq \pi$. To this end note that for $r \in[0,1)$ and $0<t \leqq \pi$ we have $-\pi / 2 \leqq \operatorname{Arg}\left(1 /\left(1-r e^{-i t}\right)\right) \leqq 0$, where Arg denotes here (and elsewhere in this paper) the principal argument. Hence we have $-(\pi / 4) \alpha \leqq$ $(\pi / 4) \alpha+\alpha \operatorname{Arg}\left(1 /\left(1-r e^{-i t}\right)\right) \leqq(\pi / 4) \alpha$. Using this fact, the fact that $0<\alpha<2$ and the identity

$$
\operatorname{Re} \frac{e^{\imath \operatorname{sgn}(t)(\pi \alpha / 4)}}{\left(1-r e^{-i t}\right)^{\alpha}}=\frac{\cos \left(\alpha(\pi / 4)+\alpha \operatorname{Arg}\left(1 /\left(1-r e^{-i t}\right)\right)\right)}{\left|1-r e^{-i t}\right|^{\alpha}}
$$

we obtain (10).

In Theorem 1 to follow we prove that Theorem $\mathrm{A}$ is sharp when $0 \leqq \alpha<2$.

THEOREM 1. Suppose $0 \leqq \alpha<2$ and $\mu$ is a complex measure on $[-\pi, \pi]$. Then there exists a function $f_{\nu} \in \mathscr{I}_{\alpha}$ such that $|\nu|=|\mu|$ and $a$ constant $A_{\alpha}$ depending only on $\alpha$ such that

$$
\left|f_{\nu}(r)\right| \geqq A_{\alpha}\left[\|\mu\|+\int_{1-r}^{\pi} \frac{w(x)}{x^{\alpha+1}} d x\right]
$$

where $w(x)=w(x, 1, \mu)$ and $r \in[0,1)$.

Proof. We suppose $\alpha>0$. The proof for $\alpha=0$ is similar and we do not 
give it here. For a complex measure $\mu$ on $[-\pi, \pi]$ define a measure $\nu$ by $d \nu(t)=e^{i \operatorname{sgn}(t)(\pi \alpha / 4)} d|\mu|(t)$ and, consequently,

$$
f_{\nu}(z)=\int_{-\pi}^{\pi} \frac{d \nu(t)}{\left(1-e^{-i t} z\right)^{\alpha}}
$$

for $z \in \Delta$. Note that $\|\nu\|=\|\mu\|$. It follows from (12) that

$$
\left|f_{\nu}(r)\right| \geqq \operatorname{Re} f_{\nu}(r)=\int_{-\pi}^{\pi} \operatorname{Re}\left[\frac{e^{2 \operatorname{sgn}(t)(\pi \alpha / 4)}}{\left(1-r e^{-i t}\right)^{\alpha}}\right] d|\mu|(t) .
$$

We infer from (4), (10) and (13) that (11) holds for $A_{\alpha}=\left(\min \left(\alpha, \pi^{-\alpha}\right) / 2^{\alpha / 2}\right) \cos$ $(\pi \alpha / 4)$.

Remark. Theorem 1 shows that for $0 \leqq \alpha<2$, Theorem A in [1] is sharp in the most strict possible manner. For $\alpha>2$ we are not able to do quite as well. The case $\alpha=2$ (and also $\alpha=6,10,14, \cdots$ ) remains open.

LEMMA 2. For any $\varepsilon>0$ there are positive constants $a, b$, and $T$ such that $T \leqq \pi$ and for all $r \in[0,1)$ we have

$$
\left|\operatorname{Arg} \frac{1}{\left(1-r e^{-i t}\right)}\right|<\varepsilon
$$

whenever $0 \leqq t \leqq a(1-r)$ and

$$
\left|\operatorname{Arg} \frac{1}{\left(1-r e^{-i t}\right)}+\frac{\pi}{2}\right|<\varepsilon
$$

whenever $b(1-r) \leqq t \leqq T$.

Proof. To verify the first inequality we note that without loss of generality we may assume that $\varepsilon<\tan ^{-1}(\pi / 2)$. Let $a=\tan \varepsilon$. Then for $0 \leqq t \leqq a(1-r)$ we have

$$
\begin{aligned}
& 0 \leqq \tan \left(-\operatorname{Arg} \frac{1}{\left(1-r e^{-i t}\right)}\right)=\frac{r \sin t}{1-r \cos t} \leqq \frac{r t}{1-r} \\
& \leqq r a<a=\tan \varepsilon,
\end{aligned}
$$

which gives (14).

We may assume without loss of generality that $\varepsilon<(\pi / 2)$ and is such that $A=\tan (\pi / 2-\varepsilon)>1$. Let $b=2 \pi A$ and $T=(2 / b)$. Then we have $T=1 / \pi A$. Noting that $b \geqq 2 \pi$ and $T<\pi$ we see that the set of $t$ 's such that $b(1-r) \leqq t \leqq T$ when $0 \leqq r<1 / 2$ is vacuous. So to prove (15) we may assume $r \in[1 / 2,1)$. The inequalities $b(1-r) \leqq t \leqq T$ are equivalent to $2 \pi A(1-r) \leqq t \leqq(1 / \pi A)$, which implies $(1-r) / t<1 / 2 \pi A$ and $r t / 2<1 / 2 \pi A$. Adding the last two inequalities we obtain $(1-r) / t+r t / 2<1 / \pi A$. This can be rewritten as $A<1 / \pi\left(t / 1-r+\left(r t^{2} / 2\right)\right)$. Since $1 / 2 \leqq r<1$ we deduce that 
(16) $\tan \left(\frac{\pi}{2}-\varepsilon\right)=A<\frac{2 t}{\pi} \frac{r}{1-r\left(1-\left(t^{2} / 2\right)\right)} \leqq \frac{2 t}{\pi} \frac{r}{1-r \cos t}<\frac{r \sin t}{1-r \cos t}$.

It is clear that (15) follows from (16).

LEMMA 3. Let $\alpha>0$ with $(\alpha+2) / 4 \notin \boldsymbol{Z}$. Let $\beta=((\pi / 2) \alpha-2 k \pi) / 2$, where $k$ is the greatest integer less than or equal to $(\alpha+2) / 4$. Then there exist positive constants $a, b, T, 0<T<\pi$, and $c_{1}$ such that

$$
\operatorname{Re} \frac{e^{-i \beta}}{\left(1-r e^{-i t}\right)^{\alpha}} \geqq \frac{c_{1}}{\left|1-r e^{-i t}\right|^{\alpha}}
$$

for every $r \in[0,1)$ and every $t$ such that $0 \leqq t \leqq a(1-r)$ or $b(1-r) \leqq t \leqq T$ or $t=\pi$.

Proof. By the definition of $\beta$ it follows that $\beta \in(-\pi / 2, \pi / 2)$. Choose $\varepsilon>0$ such that $[\beta-\alpha \varepsilon, \beta+\alpha \varepsilon] \subset(-\pi / 2, \pi / 2)$. Let $a, b$ and $T$ be such that Lemma 2 holds. We have the equality

$$
\operatorname{Re} \frac{e^{-\imath, \beta}}{\left(1-r e^{-i t}\right)^{\alpha}}=\frac{\cos \left(\beta-\alpha \operatorname{Arg}\left(1 /\left(1-r e^{-i t}\right)\right)\right)}{\left|1-r e^{-i t}\right|^{\alpha}} .
$$

Suppose $0 \leqq t \leqq a(1-r)$. Then (14) gives

$$
-\frac{\pi}{2}<\beta-\alpha \varepsilon<\beta-\alpha \operatorname{Arg} \frac{1}{\left(1-r e^{-i t}\right)}<\beta+\alpha \varepsilon<\frac{\pi}{2} .
$$

Let $d=\max \{|\beta-\alpha \varepsilon|,|\beta+\alpha \varepsilon|\}$ and note that $-\pi / 2<d<\pi / 2$. Therefore (18) and (19) imply (17) with $c_{1}=\cos d$.

Now suppose $b(1-r) \leqq t \leqq T$. Then (15) implies

$$
-\frac{\pi}{2} \alpha-\alpha \varepsilon<\alpha \operatorname{Arg} \frac{1}{\left(1-r e^{-i t}\right)}<-\frac{\pi}{2} \alpha+\alpha \varepsilon .
$$

Using the definition of $\beta$ and a short computation, (20) gives

$$
\beta-\alpha \varepsilon<\alpha \operatorname{Arg} \frac{1}{\left(1-r e^{-i t}\right)}-\beta-2 k \pi<\beta+\alpha \varepsilon .
$$

Again (18) and (21) give (17) with $c_{1}=\cos d$.

Finally, if $t=\pi$ then, since $\cos \beta>\cos d$, (17) holds also in this case. Then

LEMMA 4. Suppose $0 \leqq r<1,0 \leqq r \leqq \pi, 0<a<1<b$ and $a(1-r)<\tau<b(1-r)$.

$$
\frac{\tau}{b} \leqq\left|1-r e^{-i t}\right| \leqq \frac{2}{a} \tau
$$

whenever $0 \leqq t \leqq \tau$ and

$$
\frac{1}{2}\left|1-e^{i t}\right| \leqq\left|1-r e^{-i t}\right| \leqq 2\left|1-e^{i t}\right|
$$

whenever $t \geqq(\pi / a) \tau$. 
Proof. We note that if $0 \leqq t \leqq \tau$ then

$$
1-r \leqq\left|1-r e^{-i t}\right| \leqq(1-r)+t \leqq \frac{\tau}{a}+\tau \leqq \frac{2}{a} \tau
$$

and (24) clearly implies (22) since $\tau / b<1-r$. By the inequality $\sin x \geqq(2 / \pi) x$, $0 \leqq x<\pi / 2$, we have

$$
\frac{1}{2}\left|1-e^{i t}\right|=\sin \frac{t}{2} \geqq \frac{t}{\pi} .
$$

When $t \geqq(\pi / a) \tau$, (25) implies

$$
\frac{1}{2}\left|1-e^{i \iota}\right| \geqq \frac{\tau}{a}
$$

Hence

$$
\begin{aligned}
\left|1-r e^{-i t}\right| & =\left|e^{i t}-r\right| \geqq\left|e^{i t}-1\right|-(1-r) \\
& \geqq\left|e^{i t}-1\right|-\frac{\tau}{a} \geqq \frac{1}{2}\left|e^{i t}-1\right| .
\end{aligned}
$$

It also follows from (26) that

$$
\begin{aligned}
\left|1-r e^{-i t}\right| & =\left|e^{i t}-r\right| \leqq\left|e^{i t}-1\right|+(1-r) \\
& \leqq\left|e^{i t}-1\right|+\frac{\tau}{a} \leqq\left|e^{i t}-1\right|+\frac{1}{2}\left|e^{i t}-1\right| \\
& <2\left|e^{i t}-1\right| .
\end{aligned}
$$

So (23) holds.

We remark that in this paper we assume the notation $\sum_{l=k+1}^{l=\infty} a_{l}=\sum_{l=k+1}^{\infty} \alpha_{l}$ $+a_{\infty}$.

LEMMA 5. Let $\alpha>0$. Then for each positive sufficiently small number $q$ there is an $\eta>0$ so that for each sequence $\mu_{0}, \cdots, \mu_{k}, \cdots, \mu_{\infty}$ of non-negative numbers with $\sum_{l=0}^{l=\infty} \mu_{l}<+\infty$ there are sequences $\nu_{0}, \nu_{1}, \cdots, \nu_{k}, \cdots, \nu_{\infty}$ of nonnegative numbers, and $\tau_{0}, \tau_{1}, \cdots, \tau_{k}, \cdots, \tau_{\infty}$ satisf $y i n g$

$$
\nu_{0}=\mu_{0}, \quad \text { and } \quad \eta^{2} \sum_{l=k}^{l=\infty} \mu_{l} \leqq \sum_{l=k}^{l=\infty} \nu_{l} \leqq \sum_{l=k}^{l=\infty} \mu_{l}, \quad k=0,1, \cdots
$$

and

$$
\tau_{0}=\pi, \tau_{\infty}=0, \tau_{k}=q^{2 k} \pi \quad \text { or } \quad q^{2 k-1} \pi, \quad k=1,2, \cdots,
$$

such that for each $k \geqq 1$ we have either

$$
\frac{a^{\alpha} \nu_{k}}{2^{\alpha} \tau_{k}^{\alpha}} \geqq 2\left[2^{\alpha} \sum_{l=0}^{k-1} \frac{\nu_{l}}{\left|1-e^{-i \imath_{l}}\right|^{\alpha}}+\frac{b^{\alpha}}{\tau_{k}^{\alpha}} \sum_{l=k+1}^{l=\infty} \nu_{l}\right]
$$

or

$$
\frac{b^{\alpha} \nu_{k}}{\tau_{k}^{\alpha}} \leqq \frac{c_{1}}{2} 2^{-\alpha}\left[\sum_{l=0}^{k-1} \frac{\nu_{l}}{\left|1-e^{-\imath \tau_{l}}\right|^{\alpha}}+\frac{a^{\alpha}}{\tau_{k}^{\alpha}} \sum_{l=k+1}^{l=\infty} \nu_{l}\right]
$$


where $a, b$ and $c_{1}$ are numbers from Lemma 3.

Proof. Let $q$ be any positive number such that

$$
\frac{2^{\alpha+1}\left(2^{\alpha}+b^{\alpha}\right) q^{\alpha} b^{\alpha}}{a^{\alpha}}<c_{1} \frac{2^{-\alpha}}{2} .
$$

We introduce the auxiliary sequence $\left\{\theta_{k}\right\}$ defined by

$$
\sum_{l=k}^{l=\infty} \nu_{l}=\theta_{k} \sum_{l=k}^{l=\infty} \mu_{l}, \quad k=0,1, \cdots .
$$

In the case when $\sum_{l=k}^{l=\infty} \mu_{l}=0$ we put $\theta_{k}=1$. Conditions (27), (29) and (30) may by rewritten as the following conditions on $\left(\theta_{k}\right)$

$$
\begin{gathered}
\eta^{2} \leqq \theta_{k} \leqq 1, \quad k=0,1, \cdots, \quad \theta_{0}=\theta_{1}=1, \\
\frac{a^{\alpha} \nu_{k}}{2^{\alpha} \tau_{k}^{\alpha}} \geqq 2\left[2^{\alpha} \sum_{l=0}^{k-1} \frac{\nu_{l}}{\left|1-e^{-\imath \tau}\right|^{\alpha}}+\frac{b^{\alpha}}{\tau_{k}^{\alpha}} \theta_{k+1} \sum_{l=k+1}^{l=\infty} \mu_{l}\right]
\end{gathered}
$$

and

$$
\frac{b^{\alpha} \nu_{k}}{\tau_{k}^{\alpha}} \leqq \frac{c_{1}}{2} 2^{-\alpha}\left[\sum_{l=0}^{k-1} \frac{\nu_{l}}{\left|1-e^{-2 \tau_{l}}\right|^{\alpha}}+\frac{\alpha^{\alpha}}{\tau_{k}^{\alpha}} \theta_{k+1} \sum_{l=k+1}^{l=\infty} \mu_{l}\right]
$$

with

$$
\nu_{k}=\theta_{k} \sum_{l=k}^{l=\infty} \mu_{l}-\theta_{k+1} \sum_{l=k+1}^{l=\infty} \mu_{l} \geqq 0, k=0,1, \cdots \text { and with } \nu_{\infty}=0 \text { if } \mu_{\infty}=0 .
$$

If $\mu_{\infty}>0$ then (35) implies $\sum_{k=0}^{\infty}\left(\theta_{k}-\theta_{k+1}\right)^{-} \leqq\left(1 / \mu_{\infty}\right) \sum_{k=0}^{\infty} \mu_{k}$. This fact together with the inequality $\sum_{k=0}^{\infty}\left(\theta_{k}-\theta_{k+1}\right)^{+} \leqq \sum_{k=0}^{\infty}\left(\theta_{k}-\theta_{k+1}\right)^{-}+\sup _{k} \theta_{k}-\inf _{k} \theta_{k}$ implies $\sum_{k=0}^{\infty}\left|\theta_{k}-\theta_{k+1}\right|<+\infty$. Therefore $\lim _{k \rightarrow \infty} \theta_{k}$ exists and we define $\nu_{\infty}=\left(\lim _{k \rightarrow \infty} \theta_{k}\right)$ $\mu_{\infty}$ in this case.

We will construct sequences $\left(\theta_{k}\right)$ and $\left(\tau_{k}\right)$ inductively. To start this induction set $\theta_{0}=\theta_{1}=1, \tau_{0}=\pi$. Next suppose that $n \geqq 1$ and that $\theta_{0}, \cdots, \theta_{n}$ and $\tau_{0}$, $\cdots, \tau_{n-1}$ are already selected so that (28), (32), (33) or (34), and (35) hold for $k=1, \cdots, n-1$. If (33) or (34) is satisfied with $k=n, \tau_{n}=q^{2 n} \pi$, and $\theta_{n+1}=\theta_{n}$; then, naturally, set $\tau_{n}=q^{2 n} \pi$ and $\theta_{n+1}=\theta_{n}$. Then $\nu_{n}=\theta_{n} \mu_{n} \geqq 0$. Now assume that this is not the case, i.e., we have

$$
\frac{a^{\alpha} \theta_{n} \mu_{n}}{2^{\alpha}\left(q^{2 n} \pi\right)^{\alpha}}<2\left[2^{\alpha} \sum_{l=0}^{n-1} \frac{\nu_{l}}{\left|1-e^{-\imath \tau}\right|^{\alpha}}+\frac{b^{\alpha} \theta_{n}}{\left(q^{2 n} \pi\right)^{\alpha}} \sum_{l=n+1}^{l=\infty} \mu_{l}\right]
$$

and

$$
\frac{b^{\alpha} \theta_{n} \mu_{n}}{\left(q^{2 n} \pi\right)^{\alpha}}>\frac{c_{1}}{2} 2^{-\alpha}\left[\sum_{l=0}^{n-1} \frac{\nu_{l}}{\left|1-e^{-\imath \tau}\right|^{\alpha}}+\frac{a^{\alpha} \theta_{n}}{\left(q^{2 n} \pi\right)^{\alpha}} \sum_{l=n+1}^{l=\infty} \mu_{l}\right] .
$$

We now consider 4 cases.

Case 1. If 


$$
\sum_{l=0}^{n-1} \frac{\nu_{l}}{\left|1-e^{-\imath \tau}\right|^{\alpha}} \geqq \frac{\theta_{n}}{\left(q^{2 n} \pi\right)^{\alpha}} \sum_{l=n+1}^{l=\infty} \mu_{l},
$$

then set $\tau_{n}=q^{2 n-1} \pi, \theta_{n+1}=\theta_{n}$, and, consequently, $\nu_{n}=\theta_{n} \mu_{n}$. Then (38) combined with (36) gives

$$
\frac{a^{\alpha} \nu_{n}}{2^{\alpha} \tau_{n}^{\alpha} q^{\alpha}}<2\left[\left(2^{\alpha}+b^{\alpha}\right) \sum_{l=0}^{n-1} \frac{\nu_{l}}{\left|1-e^{-\imath \tau}\right|^{\alpha}}\right]
$$

which can be rewritten as

$$
\frac{b^{\alpha} \nu_{n}}{\tau_{n}^{\alpha}}<\frac{2^{\alpha+1}\left(2^{\alpha}+b^{\alpha}\right) q^{\alpha} b^{\alpha}}{a^{\alpha}} \sum_{l=0}^{n-1} \frac{\nu_{l}}{\left|1-e^{-\imath \tau}\right|^{\alpha}} .
$$

Clearly, by our choice of $q$, (31) and (39) imply (34) with $k=n$.

Case 2. If (38) does not hold and $1 \geqq \theta_{n}>\eta$, then we set $\tau_{n}=q^{2 n} \pi$ and

$$
\theta_{n+1}=\eta \theta_{n} \text {. }
$$

Note that (40) gives $\eta^{2}<\eta \theta_{n}=\theta_{n+1} \leqq \eta<1$ and that

$$
\nu_{n}=\theta_{n} \sum_{l=n}^{l=\infty} \mu_{l}-\eta \theta_{n} \sum_{l=n+1}^{l=\infty} \mu_{l} \geqq \theta_{n} \mu_{n} \geqq 0 .
$$

Combining the negation of (38) with (37) we obtain

$$
\frac{b^{\alpha} \theta_{n} \mu_{n}}{\left(q^{2 n} \pi\right)^{\alpha}}>\frac{c_{1}}{2} 2^{-\alpha}\left(1+a^{\alpha}\right) \frac{\theta_{n}}{\left(q^{2 n} \pi\right)^{\alpha}} \sum_{l=n+1}^{l=\infty} \mu_{l} .
$$

Inequality (42), together with (40) and (41) implies

$$
\frac{a^{\alpha} \nu_{n}}{\tau_{n}^{\alpha}}>\frac{1}{\eta} \frac{a^{\alpha}\left(1+a^{\alpha}\right) c_{1}}{2 \cdot 2^{\alpha} b^{2 \alpha}} \frac{b^{\alpha} \theta_{n+1}}{\tau_{n}^{\alpha}} \sum_{l=n+1}^{l=\infty} \mu_{l}
$$

Clearly (43) implies (33) (with $k=n$ ) if only $\eta$ is sufficiently small.

Case 3. If (38) does not hold, $\theta_{n} \leqq \eta$ and

$$
\frac{1}{\eta} \sum_{l=n+1}^{l=\infty} \mu_{l}<\sum_{l=n}^{l=\infty} \mu_{l}
$$

then we set $\tau_{n}=q^{2 n} \pi$, and $\theta_{n+1}=(1 / \eta) \theta_{n}$. Note that $\eta^{2} \leqq\left(\theta_{n} / \eta\right)=\theta_{n+1} \leqq 1$. Moreover, by (44), we have $\nu_{n}=\theta_{n} \sum_{l=n}^{l=\infty} \mu_{l}-\left(\theta_{n} / \eta\right) \sum_{l=n+1}^{l=\infty} \mu_{l} \geqq 0$. Also since we may require that $\eta<1$, we have $\nu_{n}=\theta_{n} \mu_{n}+\theta_{n}(1-(1 / \eta)) \sum_{l=n+1}^{l=\infty} \mu_{l} \leqq \theta_{n} \mu_{n}$. This last inequality together with the negation of (38) applied to (36) yields

$$
\frac{a^{\alpha} \nu_{n}}{2^{\alpha} \tau_{n}^{\alpha}} \leqq 2 \eta\left(2^{\alpha}+b^{\alpha}\right) \frac{\theta_{n+1}}{\tau_{n}^{\alpha}} \sum_{l=n+1}^{l+\infty} \mu .
$$

Inequality (45) may be rewritten as 


$$
\frac{b^{\alpha} \nu_{n}}{\tau_{n}^{\alpha}} \leqq 2^{2} \eta \frac{2^{\alpha} b^{\alpha}\left(2^{\alpha}+b^{\alpha}\right)}{a^{2 \alpha} c_{1}} \frac{a^{\alpha} c_{1}}{2 \cdot 2^{\alpha}} \frac{\theta_{n+1}}{\tau_{n}^{\alpha}} \sum_{l=n+1}^{l=\infty} \mu_{l}
$$

which clearly implies (34) with $k=n$ if $\eta$ is sufficiently small.

Case 4. It only remains to deal with the case when $\theta_{n} \leqq \eta$ and neither (38) nor (44) hold. In this case put

$$
\tau_{n}=q^{2 n} \pi \quad \text { and } \quad \theta_{n+1}=\frac{\sum_{l=n}^{l=\infty} \mu_{l}}{\sum_{l=n+1}^{l=\infty} \mu_{1}} \theta_{n} .
$$

Since (44) is not true we have $\eta^{2} \leqq \theta_{n} \leqq \theta_{n+1} \leqq(1 / \eta) \theta_{n} \leqq(\eta / \eta)=1$. A simple calculation shows that $\nu_{n}=0$ and so (34) is trivially satisfied.

LEMMA 6. Suppose that $\alpha>0$ is such that $(\alpha+2) / 4$ is not an integer. Let $c_{1}$ be the constant from Lemma 3 , and let $a, b$, and $T$ be the constants from Lemma 3. Let $0<q<\min (a / b, T / \pi, a / \pi)$. Then there is a constant $c_{2}>0$ such that for any sequences: ( $\tau_{k}$ satisfying (28) of Lemma 5 , and non-negative $\nu_{0}, \cdots, \nu_{k}, \cdots$, $\nu_{\infty}$ which for each $k, k=1,2, \cdots$, satisfies either (29) or (30) of Lemma 5 we have

$$
\left|\sum_{l=0}^{l=\infty} \frac{\nu_{l}}{\left(1-r e^{-\imath \tau} l\right)^{\alpha}}\right| \geqq c_{2} \sum_{l=0}^{l=\infty} \frac{\nu_{l}}{\left|1-r e^{-\imath \tau}\right|^{\alpha}},
$$

for $0 \leqq r \leqq 1$.

Proof. Note that for any positive integer $k$ the inequality (29) of Lemma 5 implies that

$$
\frac{\nu_{k}}{\left|1-r e^{-\imath \tau_{k}}\right|^{\alpha}} \geqq 2 \sum_{l \neq k} \frac{\nu_{l}}{\left|1-r e^{-\imath \tau_{l}}\right|^{\alpha}},
$$

when $a(1-r)<\tau_{k}<b(1-r)$, while the inequality (30) of Lemma 5 implies that

$$
\frac{\nu_{k}}{\left|1-r e^{-\imath \tau_{k}}\right|^{\alpha}} \leqq \frac{c_{1}}{2} \sum_{l \neq k} \frac{\nu_{l}}{\left|1-r e^{-\imath \tau} l\right|^{\alpha}}
$$

where $a(1-r)<\tau_{k}<b(1-r)$. Indeed, since $\tau_{k} \leqq q \tau_{l} \leqq(a / \pi) \tau_{l}$, the first inequality of (23) in Lemma 4 gives

$$
2^{\alpha} \sum_{l=0}^{k-1} \frac{\nu_{l}}{\left|1-e^{-i \tau}\right|^{\alpha}} \geqq \sum_{l=0}^{k-1} \frac{\nu_{l}}{\left|1-r e^{-\imath \tau}\right|^{\alpha}} \geqq 2^{-\alpha} \sum_{l=0}^{k-1} \frac{\nu_{l}}{\left|1-e^{-\imath \tau}\right|^{\alpha}} .
$$

Then, since $\tau_{k}<b(1-r)$ we have

$$
\frac{b^{\alpha}}{\tau_{k}^{\alpha}} \sum_{l=k+1}^{l=\infty} \nu_{l} \geqq \sum_{l=k+1}^{l=\infty} \frac{\nu_{l}}{\left|1-r e^{-\imath \tau}\right|^{\alpha}} \geqq \frac{2^{-\alpha} a^{\alpha}}{\tau_{k}^{\alpha}} \sum_{l=k+1}^{l=\infty} \nu_{l} .
$$

Finally, applying the second inequality of (22) Lemma 4 we get 


$$
\frac{a^{\alpha} \nu_{k}}{2^{\alpha} \tau_{k}^{\alpha}} \leqq \frac{\nu_{k}}{\left|1-r e^{-\imath \tau_{k}}\right|^{\alpha}} \leqq \frac{b^{\alpha} \nu_{k}}{\tau_{k}^{\alpha}}
$$

Combining (49), (50) and (51) with (29) we obtain (47). The proof of (48) which we do not give here can be given in a similar fashion. If $\left\{\tau_{k}\right\}_{k=1}^{k=\infty} \cap$ $(a(1-r), b(1-r))=\emptyset$, then since $\tau_{1} \leqq q \pi \leqq T$ we have $\left\{\tau_{k}\right\}_{k=0}^{k=\infty} \subset[0, a(1-r)] \cup$ $[b(1-r), T] \cup\{\pi\}$. Then, by Lemma 3 we have

$$
\left|\sum_{l=0}^{l=\infty} \frac{\nu_{l}}{\left(1-r e^{-i \tau}\right)^{\alpha}}\right| \geqq \sum_{l=0}^{l=\infty} \nu_{l} \operatorname{Re} \frac{e^{-i \beta}}{\left(1-r e^{-i \imath}\right)^{\alpha}} \geqq c_{1} \sum_{l=0}^{l=\infty} \frac{\nu_{l}}{\left|1-r e^{-\imath \tau}\right|^{\alpha}} .
$$

with $\beta$ from Lemma 3 . If this is not the case, then since $\left(\tau_{l+1} / \tau_{l}\right) \leqq q<(a / b)$, $l=0,1, \cdots$, we have $a(1-r)<\tau_{k}<b(1-r)$ for exactly one $k, k \geqq 1$. By what was proved earlier, either (47) or (48) holds for this case. If (47) holds then we have

$$
\begin{aligned}
\left|\sum_{l=0}^{l=\infty} \frac{\nu_{l}}{\left(1-r e^{-2 \tau} l\right)^{\alpha}}\right| & \geqq \frac{\nu_{k}}{\left|1-r e^{-\imath \tau_{k}}\right|^{\alpha}}-\sum_{l \neq k} \frac{\nu_{l}}{\left|1-r e^{-\imath \tau}\right|^{\alpha}} \\
& \geqq \frac{1}{3} \sum_{l=0}^{l=\infty} \frac{\nu_{l}}{\left|1-r e^{-\imath \tau}\right|^{\alpha}} .
\end{aligned}
$$

If (48) holds, then, since all terms of $\left(\tau_{l}\right)$ except for $\tau_{k}$ are in $[0, a(1-r)] \cup$ $[b(1-r), T] \cup\{\pi\}$, applying Lemma 3 and the fact that $0<c_{1}<1$ we get

$$
\begin{aligned}
\left|\sum_{l=0}^{l=\infty} \frac{\nu_{l}}{\left(1-r e^{-\imath \tau} l\right)^{\alpha}}\right| & \geqq\left|\sum_{l \neq k} \frac{\nu_{l}}{\left(1-r e^{-\imath \tau} l\right)^{\alpha}}\right|-\frac{\nu_{k}}{\left|1-r e^{-\imath \tau_{k}}\right|^{\alpha}} \\
& \geqq \sum_{l \neq k} \nu_{l} \operatorname{Re} \frac{e^{-i \beta}}{\left(1-r e^{-\imath \tau}\right)^{\alpha}}-\frac{\nu_{k}}{\left|1-r e^{-\imath \tau}\right|^{\alpha}} \\
& \geqq c_{1} \sum_{l \neq k} \frac{\nu_{l}}{\left|1-r e^{-\imath \tau}\right|^{\alpha}}-\frac{\nu_{k}}{\left|1-r e^{-i \tau_{k}}\right|^{\alpha}} \\
& \geqq \frac{c_{1}}{3} \sum_{l=0}^{l=\infty} \frac{\nu_{l}}{\left|1-r e^{-\imath \tau}\right|^{\alpha}}
\end{aligned}
$$

which completes the proof.

Before we state Theorem 2 we recall the notation

$$
w(t, \nu)=\left\{\begin{array}{l}
0 \quad t=0 \\
|\nu|([-t, t]) \quad 0<t \leqq \pi
\end{array}\right.
$$

and likewise for $\mu$. Note that this implies

$$
\int_{0}^{\pi} f(t) d w(t, \nu)=\int_{-\pi}^{\pi} f(|t|) d|\nu|(t)
$$

for each $f$ continuous on $[0, \pi]$.

THEOREM 2. For each $\alpha>2$ with $(\alpha+2) / 4$ not an integer there is a positive 
constant $c_{3}$ depending only on $\alpha$ such that for any measure $\mu$ on $[-\pi, \pi]$ there is a measure $\nu$ on $[0, \pi]$ such that

$$
w(t, \nu) \leqq w(t, \mu)
$$

for $0 \leqq t \leqq \pi$, and

$$
\left|f_{\nu}(r)\right| \geqq c_{3} \int_{-\pi}^{\pi} \frac{d|\mu|(t)}{\left|1-r e^{-i t}\right|^{\alpha}}
$$

for $r \in[0,1)$ where $f_{\nu}$ is defined by (1).

Remark. Note that by Proposition 1, the right-hand side of (53) in the theorem may be replaced by

$$
c_{3}\left[\|\mu\|+\int_{1-r}^{\pi} \frac{w(t, \mu)}{t^{\alpha+1}} d t\right]
$$

and so Theorem 2 proves that Theorem $\mathrm{A}$ in [1] is sharp.

Proof. Without loss of generality we may and so assume that $\mu$ is a nonnegative measure with support contained in $[0, \pi]$. Let a sequence $\mu_{0}, \mu_{1}, \cdots$, $\mu_{\infty}$ be defined by the formula

$$
\mu_{k}=\mu\left(\left(q^{2 k+2} \pi, q^{2 k} \pi\right]\right), \quad k \geqq 0
$$

and $\mu_{\infty}=\mu(\{0\})$, where $q$ is a positive number for which both Lemma 5 and Lemma 6 hold. Note that $q$ depends only on $\alpha$. Let $\left(\tau_{k}\right)$ and $\left(\nu_{k}\right)$ be sequences constructed in Lemma 5 for this $q$. Define measures $\tilde{\mu}$ and $\nu$ by the formulas

$$
\tilde{\mu}=\sum_{l=0}^{l=\infty} \mu_{l} \delta_{\tau_{l}}
$$

and

$$
\nu=\sum_{l=0}^{l=\infty} \nu_{l} \delta_{\tau_{l}} .
$$

Since the measure $\tilde{\mu}$ is obtained by "sweeping" the mass from the interval $\left(q^{2 k+2} \pi, q^{2 k} \pi\right]$ to the point $\tau_{k}(k=0,1, \cdots)$, which is given by (28) of Lemma 5 , and which is located to the right of the interval, without moving the mass concentrated at 0 and at $\pi$ we have

$$
w(t, \tilde{\mu}) \leqq w(t, \mu)
$$

for $0 \leqq t \leqq \pi$. Moreover, since $\left|1-r e^{-\imath s t}\right| \leqq s\left|1-r e^{-i t}\right|$ for $0<t, s>1$, st $<\pi$ and $r \in[0,1)$, we have

$$
\begin{aligned}
\int_{[0, \pi]} \frac{d \mu(t)}{\left|1-r e^{-i t}\right|^{\alpha}} & =\frac{\mu_{\infty}}{(1-r)^{\alpha}}+\sum_{k=0}^{\infty} \int_{\left(q^{\left.2 k+2 \pi, q^{2 k} \pi\right]}\right.} \frac{d \mu(t)}{\mid 1-r e^{-\left.i t\right|^{\alpha}}} \\
& \leqq \frac{\mu_{\infty}}{(1-r)^{\alpha}}+\sum_{k=0}^{\infty} \int_{\left(q^{\left.2 k+2 \pi, q^{2 k} \pi\right]}\right.} \frac{d \mu(t)}{\mid 1-r e^{-\left.\imath q^{2 k+2 \pi}\right|^{\alpha}}}
\end{aligned}
$$




$$
\begin{aligned}
& =\frac{\mu_{\infty}}{(1-r)^{\alpha}}+\sum_{k=0}^{\infty} \frac{\mu_{k}}{\mid 1-r e^{-\left.2 q^{2 k+2 \pi}\right|^{\alpha}}} \\
& =\frac{\mu_{\infty}}{(1-r)^{\alpha}}+\sum_{k=0}^{\infty}\left(\frac{\tau_{k}}{q^{2 k+2} \pi}\right)^{\alpha} \frac{\mu_{k}}{\left|1-r e^{-2 \tau_{k}}\right|^{\alpha}} \\
& \leqq q^{-3 \alpha} \frac{\mu_{\infty}}{(1-r)^{\alpha}}+q^{-3 \alpha} \sum_{k=0}^{\infty} \frac{\mu_{k}}{\mid 1-r e^{-\left.i \tau_{k}\right|^{\alpha}}} \\
& =q^{-3 \alpha} \int_{[0, \pi]} \frac{d \tilde{\mu}(t)}{\left|1-r e^{-i t}\right|^{\alpha}} .
\end{aligned}
$$

Note that (54) and the second inequality in (27) of Lemma 5 give (52).

We next prove that

$$
\int_{[0, \pi]} \frac{d \nu(t)}{\left|1-r e^{-i t}\right|^{\alpha}} \geqq \frac{\eta^{2} q^{3 \alpha}}{2} \int_{[0, \pi]} \frac{d \tilde{\mu}(t)}{\left|1-r e^{-i t}\right|^{\alpha}}
$$

for $0 \leqq r<1$. First note, it follows from (27), the choice of $\tau_{k}$ and the fact that $w(t, \tilde{\mu})$ is nondecreasing that $w(t, \nu) \geqq \eta^{2} w\left(q^{3} t, \tilde{\mu}\right)$ for $0 \leqq t<\pi$ and $w(\pi, \nu) \geqq \eta^{2} w(\pi$, $\tilde{\mu})$. Using the foregoing facts, the inequality $\left(1 /\left|1-r e^{-\imath q^{-3} t}\right|^{\alpha}\right) \geqq q^{3 \alpha}\left(1 /\left|1-r e^{-i t}\right|^{\alpha}\right)$ whenever $0 \leqq t \leqq q^{3} \pi$ and the fact that $1 /\left|1-r e^{-\imath q^{3} t}\right|^{\alpha}$ is nondecreasing, it is readily proved that

$$
\int_{0}^{\pi} \frac{d w(t, \nu)}{\left|1-r e^{-i t}\right|^{\alpha}} \geqq \eta^{2} \int_{0}^{\pi} \frac{d w\left(q^{3} t, \tilde{\mu}\right)}{\left|1-r e^{-i t}\right|^{\alpha}}=\eta^{2} \int_{0}^{q^{3 \pi}} \frac{d w(t, \tilde{\mu})}{\left|1-r e^{-\imath q^{-3} t}\right|^{\alpha}} \geqq \eta^{2} q^{3 \alpha} \int_{0}^{q^{3 \pi} \pi} \frac{d w(t, \tilde{\mu})}{\left|1-r e^{-i t}\right|^{\alpha}}
$$

and

$$
\int_{0}^{\pi} \frac{d w(t, \nu)}{\left|1-r e^{-i t}\right|^{\alpha}} \geqq \eta^{2} q^{3 \alpha} \int_{q^{3} \pi}^{\pi} \frac{d w(t, \tilde{\mu})}{\left|1-r e^{-i t}\right|^{\alpha}} .
$$

These last two inequalities imply (56).

Combining (55) and (56) with (46) of Lemma 6 we obtain (53) with $c_{3}=q^{6 \alpha} \eta^{2} c_{2} / 2$.

The following two lemmas are technical results needed for the proof of Theorem 3.

LEMMA 7. Let $\alpha>1$. Then there is $a \delta>0$ such that for each positive nondecreasing $C^{1}$ function $\tilde{\varepsilon}(t), 0<t \leqq \pi$, satısfying

$$
\frac{d \log \tilde{\varepsilon}(t)}{d \log t} \leqq \delta, \quad 0<t \leqq \pi
$$

we have

$$
|h(s)| \geqq \frac{\tilde{\varepsilon}(s)}{4 s^{\alpha-1}(\alpha-1)}
$$

for all sufficiently small $s>0$, where

$$
h(s)=\int_{0}^{\pi}(s+i t)^{-\alpha} \tilde{\varepsilon}(t) d t, \quad s>0 .
$$


Proof. Note that

$$
\int_{0}^{\infty}(1+i u)^{-\alpha} d u=\frac{-i}{\alpha-1} .
$$

Choose $u_{1}$ and $u_{2}, 0<u_{1}<1<u_{2}<+\infty$, so that

$$
\int_{0}^{u_{1}}|1+i u|^{-\alpha} d u \leqq \frac{1}{8(\alpha-1)}
$$

and

$$
u_{2}^{1-\alpha}<\frac{1}{16} .
$$

Note that (58), (59) and (60) imply that

$$
\left|\int_{u_{1}}^{u_{2}}(1+i u)^{-\alpha} d u\right| \geqq \frac{3}{4} \frac{1}{\alpha-1} .
$$

Choose $\delta$ so that $0<\delta<(\alpha-1) / 2$ and that

$$
\left(1-\left(\frac{u_{1}}{u_{2}}\right)^{\delta}\right) \int_{0}^{\infty}|1+i u|^{-\alpha} d u \leqq \frac{1}{8(\alpha-1)} .
$$

Note that (57) implies that for any $t_{0} \in(0,1)$ we have

$$
\tilde{\varepsilon}(t) \leqq\left(\frac{t}{t_{0}}\right)^{\delta} \tilde{\varepsilon}\left(t_{0}\right), \quad t_{0} \leqq t \leqq \pi,
$$

and

$$
\tilde{\varepsilon}(t) \geqq\left(\frac{t}{t_{0}}\right)^{\delta} \tilde{\varepsilon}\left(t_{0}\right), \quad 0 \leqq t \leqq t_{0} .
$$

If $s<\left(\pi / u_{2}\right)$, then we may write

$$
h(s)=s^{1-\alpha}\left[I_{1}+I_{2}+I_{3}\right]
$$

where

$$
I_{1}=\int_{0}^{u_{1}}(1+i u)^{-\alpha} \tilde{\varepsilon}(s u) d u, \quad I_{2}=\int_{u_{1}}^{u_{2}}(1+i u)^{-\alpha} \tilde{\varepsilon}(s u) d u
$$

and

$$
I_{3}=\int_{u_{2}}^{\pi / s}(1+i u)^{-\alpha} \tilde{\varepsilon}(s u) d u .
$$

Since $\tilde{\varepsilon}(t)$ is non-decreasing we obtain, by (59),

$$
\left|I_{1}\right| \leqq \int_{0}^{u_{1}}|1+i u|^{-\alpha} \tilde{\varepsilon}(s u) d u \leqq \frac{\tilde{\varepsilon}\left(s u_{2}\right)}{8(\alpha-1)} .
$$

While (63), with $t_{0}=s u_{2}$, and (60) yield 


$$
\begin{aligned}
\left|I_{3}\right| & \leqq \int_{u_{2}}^{\pi / s}|1+i u|^{-\alpha} \tilde{\varepsilon}(s u) d u \\
& \leqq \int_{u_{2}}^{\pi / s}|1+i u|^{-\alpha} \tilde{\varepsilon}\left(s u_{2}\right)\left(\frac{u}{u_{2}}\right)^{\delta} d u \\
& \leqq \int_{u_{2}}^{\infty} u^{-\alpha} \tilde{\varepsilon}\left(s u_{2}\right) u^{\delta} u_{2}^{\delta} d u \\
& =\frac{1}{\alpha-1-\delta} u_{2}^{1-\alpha} \tilde{\varepsilon}\left(s u_{2}\right) \leqq \frac{\tilde{\varepsilon}\left(s u_{2}\right)}{8(\alpha-1)} .
\end{aligned}
$$

By (64), with $t_{0}=s u_{2}$, and by (62), we have

$$
\begin{aligned}
& \left|\tilde{\varepsilon}\left(s u_{2}\right) \int_{u_{1}}^{u_{2}}(1+i u)^{-\alpha} d u-I_{2}\right| \\
& \quad=\left|\int_{u_{1}}^{u_{2}}(1+i u)^{-\alpha}\left[\tilde{\varepsilon}\left(s u_{2}\right)-\tilde{\varepsilon}(s u)\right] d u\right| \\
& \quad \leqq \int_{u_{1}}^{u_{2}}|1+i u|^{-\alpha} \tilde{\varepsilon}\left(s u_{2}\right)\left(1-\left(\frac{u_{1}}{u_{2}}\right)^{\delta}\right) d u \\
& \quad \leqq \frac{\tilde{\varepsilon}\left(s u_{2}\right)}{8(\alpha-1)} .
\end{aligned}
$$

Combining (65), (66), (67) and (68) we obtain

$$
\left|h(s)-s^{1-\alpha} \tilde{\varepsilon}\left(s u_{2}\right) \int_{u_{1}}^{u_{2}}(1+i u)^{-\alpha} d u\right| \leqq \frac{3}{8} s^{1-\alpha} \frac{\tilde{\varepsilon}\left(s u_{2}\right)}{\alpha-1} .
$$

This, together with (61) and the fact that $\tilde{\varepsilon}$ is nondecreasing gives

$$
|h(s)| \geqq \frac{3 \tilde{\varepsilon}\left(s u_{2}\right)}{8(\alpha-1) s^{\alpha-1}} \geqq \frac{\tilde{\varepsilon}(s)}{4 s^{\alpha-1}(\alpha-1)}
$$

when $0<s<\left(\pi / u_{2}\right)$.

LEMMA 8. Let $\alpha>1$. Then

$$
\lim _{s \rightarrow 0^{+}} s^{\alpha-1} \int_{0}^{\pi}\left|(s+i t)^{-\alpha}-\left(s+\left(1-e^{-i t}\right)\right)^{-\alpha}\right| d t=0 .
$$

Proof. Denote the integrand in the preceding expression by $k(s, t)$. Note that

$$
k(s, t) \leqq \frac{2}{\left|1-e^{-i t}\right|^{\alpha}} \leqq \frac{c}{t^{\alpha}}, \quad 0<t \leqq \pi
$$

for some constant $c>0$. On the other hand since $\left|(s+i t)-\left(s+\left(1-e^{-i t}\right)\right)\right| \leqq t^{2}$, $0 \leqq t \leqq \pi$, we may write

$$
k(s, t) \leqq t^{2} \sup _{z} \alpha|z|^{-\alpha-1}, \quad 0 \leqq t \leqq \pi,
$$


where the supremum is taken over the closed line segment joining $s+i t$ and $s+\left(1-e^{-i t}\right)$. If $s \leqq(1 / 4)$ and $0 \leqq t \leqq(\sqrt{s} / 2)$ then for each $z$ in this interval we have

$$
|z-(s+i t)| \leqq\left|(s+i t)-\left(s+\left(1-e^{-i t}\right)\right)\right| \leqq t^{2} \leqq \frac{1}{4} \min (t, s) .
$$

Hence for those $z$ 's we have

$$
|z|^{-\alpha-1} \leqq \begin{cases}\left(\frac{3}{4} t\right)^{-\alpha-1}, & s \leqq t \leqq \frac{\sqrt{s}}{2} \\ \left(\frac{3}{4} s\right)^{-\alpha-1}, & 0 \leqq t \leqq s .\end{cases}
$$

Combining (69), (70) and (71) we get for $0<s \leqq(1 / 4)$,

$$
k(s, t) \leqq c \begin{cases}t^{-\alpha}, & \frac{\sqrt{s}}{4} \leqq t \leqq \pi \\ t^{-\alpha+1}, & s \leqq t \leqq \frac{\sqrt{s}}{2} \\ s^{-\alpha+1}, & 0 \leqq t \leqq s .\end{cases}
$$

Applying these estimates to $\int_{0}^{\pi} k(s, t) d t$ we easily obtain the Lemma.

Our next result shows that Theorem $\mathrm{B}$ is sharp. When $1 \leqq \alpha \leqq 2$ a weaker sharpness result containing a limit superior was obtained in [2].

THEOREM 3. Let $\alpha>1$ and let $\varepsilon(r)$ be a positive function on $0 \leqq r<1$ with $\lim _{r-1}-\varepsilon(r)=0$. Then there is a differentiable function $g(t),-\pi \leqq t \leqq \pi$, so that

$$
\lim _{r \rightarrow 1^{-}} \frac{\left|\int_{-\pi}^{\pi}\left(1-r e^{-i t}\right)^{-\alpha} d g(t)\right|}{\varepsilon(r)(1-r)^{1-\alpha}}=+\infty .
$$

Proof. Denote $\tilde{\varepsilon}(t)=\sqrt{\varepsilon(1-t)}, 0<t \leqq 1$, and $\tilde{\varepsilon}(t)=\sqrt{\varepsilon(0)}, 1<t \leqq \pi$. Note that if the assertion of the theorem is true with $\varepsilon(r)=\varepsilon_{1}(r)$ and $\varepsilon_{2}(r) \leqq \varepsilon_{1}(r)$, then it is also true with $\varepsilon(r)=\varepsilon_{2}(r)$. Hence, by replacing $\varepsilon(r)$ with a larger function we may assume additionally that:

i ) $\tilde{\varepsilon}$ is $C^{1}$ and nondecreasing on $(0, \pi]$,

ii ) $\lim _{s \rightarrow 0^{+}} \frac{s^{\alpha-1}}{\tilde{\varepsilon}(s)} \int_{0}^{\pi}\left|(s+i t)^{-\alpha}-\left(s+\left(1-e^{-i t}\right)\right)^{-\alpha}\right| d t=0$,

(Lemma 8 is used to ensure this),

iii) $(d \log \tilde{\varepsilon}(t) / d \log t) \leqq \delta, 0<t \leqq \pi$, with $\delta$ being the positive constant from Lemma 7.

To obtain iii) note that for each bounded above real function $a(s)$ defined on a semifinite right-bounded interval with $\lim _{s \rightarrow-\infty} a(s)=-\infty$ there is a $C^{1}$ function $a_{1}$ defined on the same interval, and such that $\lim _{s \rightarrow-\infty} a_{1}(s)=-\infty$ and 
$\left(d a_{1}(s) / d s\right) \leqq \delta$. Then take $a(s)=\log \tilde{\varepsilon}\left(e^{s}\right)$ with $\tilde{\varepsilon}$ satisfying ii) and replace $\tilde{\varepsilon}(t)$ with $\exp \left(a_{1}(\log t)\right)$.

Let us define

$$
g(t)= \begin{cases}0, & t \leqq 0 \\ \int_{0}^{t} \tilde{\varepsilon}(u) d u, & 0 \leqq t \leqq \pi\end{cases}
$$

Clearly $g \in C^{1}[-\pi, \pi]$.

Note that

$$
f(r)=\int_{-\pi}^{\pi}\left(1-r e^{-i t}\right)^{-\alpha} d g(t)=\int_{0}^{\pi}\left(1-r e^{-i t}\right)^{-\alpha} \tilde{\varepsilon}(t) d t .
$$

Let $h(s)=\int_{0}^{\pi}(s+i t)^{-\alpha} \tilde{\varepsilon}(t) d t$. By Lemma 7 we have

$$
s^{\alpha-1}|h(s)| \geqq \frac{\tilde{\varepsilon}(s)}{4(\alpha-1)}
$$

for all sufficiently small positive $s$.

Observe now that

$$
\begin{aligned}
\left|f(r)-r^{-\alpha} h\left(\frac{1-r}{r}\right)\right| & \leqq \int_{0}^{\pi}\left|\left(1-r e^{-i t}\right)^{-\alpha}-r^{-\alpha}\left(\frac{1-r}{r}+i t\right)^{-\alpha}\right| \tilde{\varepsilon}(t) d t \\
& \leqq \tilde{\varepsilon}(\pi) r^{-\alpha} \int_{0}^{\pi}\left|\left(\frac{1-r}{r}+\left(1-e^{-i t}\right)\right)^{-\alpha}-\left(\frac{1-r}{r}+i t\right)^{-\alpha}\right| d t .
\end{aligned}
$$

By (ii) above, the last expression multiplied by $(1-r)^{\alpha-1} / \tilde{\varepsilon}((1-r)) / r$ tends to 0 when $r$ approaches 1 . Hence, by (72) with $s=(1-r) / r$, for all $r$ sufficiently close to 1 we have

$$
(1-r)^{\alpha-1}|f(r)| \geqq \frac{\tilde{\varepsilon}((1-r) / r)}{8(\alpha-1)} \geqq \frac{\tilde{\varepsilon}(1-r)}{8(\alpha-1)}=\frac{\sqrt{\varepsilon(r)}}{8(\alpha-1)}
$$

Therefore, $\quad(1-r)^{\alpha-1}|f(r)| / \varepsilon(r) \geqq 1 /(8(\alpha-1) \sqrt{\varepsilon(r)})$ for such $r$ 's. But since $\lim _{r \rightarrow 1-} \varepsilon(r)=0$, the proof is complete.

Remark. When $\alpha=1$ it is possible to prove that for any $\varepsilon(r)$ as in Theorem 3 , there is a differentiable function $g(t),-\pi \leqq t \leqq \pi$, so that

$$
\lim _{r \rightarrow 1^{-}} \frac{\left|\int_{-\pi}^{\pi} \log \left(1 /\left(1-r e^{-i t}\right)\right) d g(t)\right|}{\varepsilon(r) \log 1 /(1-r)}=+\infty .
$$

We do not give the details. Such a result with a limit superior replacing the limit was obtained in [2].

\section{REFERENCES}

[1] D. J. HALlenbeck AND T.H. MacGregor, Radial limits and radial growth of Cauchy-Stieltjes transforms, Complex Variables Theory Appl., 21 (1993), 219-229. 
[2] D. J. HAllenbeck And T.H. MacGregor, Radial growth and exceptional sets for Cauchy-Stieltjes integrals, Proc. Edinburgh Math. Soc., 37 (1993), 73-89.

[3] R.A. Hibschweiler AND T.H. MAcGregor, Closure properties of families of Cauchy-Stieltjes transforms, Proc. Amer. Math. Soc., 105 (1989), 615-621.

[4] R. A. HibschweIler AND T.H. MACGREGor, Multipliers of families of CauchyStieltjes transforms, Trans. Amer. Math. Soc., 331 (1992), 377-394.

Department of Mathematical Sciences

UNiversity of Delaware

Newark, Delaware 19716

USA

Instytut Matematyki, Politechnika Wroclawska

WYBRZEŻE ST. WYSPIAŃSKIEGO 27

50-370 WROCLAW

POLAND 